\title{
Communication \\ Suppression of Dissolution Rate via Coordination Complex in Tungsten Chemical Mechanical Planarization
}

\author{
Kangchun Lee ${ }^{1, *}$ and Jihoon Seo ${ }^{2}$ (D) \\ 1 Foundry Process Development Team, Semiconductor R\&D Center, Samsung Electronics, Samsungjeonja-ro, \\ Hwaseong 18448, Korea \\ 2 Department of Chemical and Biomolecular Engineering, Clarkson University, Potsdam, \\ New York, NY 13699, USA; jseo@clarkson.edu \\ * Correspondence: kc.lee@samsung.com
}

Citation: Lee, K.; Seo, J. Suppression of Dissolution Rate via Coordination Complex in Tungsten Chemical Mechanical Planarization. Appl. Sci. 2022, 12, 1227. https://doi.org/ 10.3390/app12031227

Academic Editor: Fethi Bedioui

Received: 3 January 2022

Accepted: 20 January 2022

Published: 24 January 2022

Publisher's Note: MDPI stays neutral with regard to jurisdictional claims in published maps and institutional affiliations.

Copyright: (C) 2022 by the authors. Licensee MDPI, Basel, Switzerland. This article is an open access article distributed under the terms and conditions of the Creative Commons Attribution (CC BY) license (https:// creativecommons.org/licenses/by/ $4.0 /)$.

\begin{abstract}
Topography of tungsten should be assured at a minimum through chemical mechanical planarization (CMP) in the metal gate structures (e.g., buried gates, replacement metal gates) and via contact in the middle of line (MOL) process for sub-7 $\mathrm{nm}$ semiconductor applications. However, excessive tungsten dissolution during the CMP process that results from high oxidizer concentrations and acidic atmospheres results in poor tungsten topography. In this study, we report a novel strategy to improve the tungsten topography by suppressing tungsten dissolution via coordination complex formations between picolinic acid and tungsten oxide. With $1.5 \mathrm{wt} \%$ picolinic acid for the inhibitor, the dissolution rate of tungsten was dramatically attenuated, and improved topography with a $\mathrm{R}_{\mathrm{a}}$ value of $7.8 \mathrm{~nm}$ were demonstrated while validating CMP removal rate.
\end{abstract}

Keywords: chemical mechanical planarization; tungsten dissolution; inhibitor; coordination complex

\section{Introduction}

Tungsten has been used as a filling material for semiconductor structures such as buried metal gates and contact via holes in the semiconductor process due to its great conductivity and gap filling ability [1-3]. However, after tungsten deposition by chemical vapor deposition (CVD), a chemical mechanical planarization (CMP) process is necessary because the inherent characteristics of tungsten result in topographic features with widths of approximately 60 to $120 \mathrm{~nm}$ and heights of 50 to $80 \mathrm{~nm}$ [1,3]. As devices evolve from the planar DRAM structures to next-generation vertical structures and design rules shrink to sub $48 \mathrm{~nm}$ contact poly pitch (CPP) in logic devices, the number of tungsten CMP processes increased from four to six times, and topography removal should be controlled to below $10 \mathrm{~nm}$ to reduce defects in subsequent processes. Therefore, a low defect buffing CMP step capable of eliminating tungsten topography with a relatively low removal rate of $100 \AA / \mathrm{min}$ is crucial. In this regard, understanding the dissolution kinetics and modulating excessive dissolution of tungsten films are necessary to achieve high surface flatness with improved topography.

Generally, in the tungsten CMP process, hydrogen peroxide $\left(\mathrm{H}_{2} \mathrm{O}_{2}\right)$ is used as an oxidant to oxidize the tungsten surface to enable a high removal rate [4-7]. However, the use of hydrogen peroxide leads to the dissolution of tungsten, which in turn adversely affects the tungsten topography. Therefore, since there is a trade-off between the removal rate and dissolution of tungsten when using hydrogen peroxide, it is necessary to develop a new approach that can both suppress dissolution and secure a high tungsten removal rate [8]. Seo et al. studied the corrosion behaviors of tungsten in the presence of hydrogen peroxide and calculated the kinetic constant [5]. Anik et al. and Kanner et al. investigated the mechanism of tungsten dissolution kinetics using Raman spectroscopy and electrochemistry $[9,10]$. Given that the tungsten film has a negative charge in an acidic CMP environment, the conventional strategies to overcome the dissolution issue focused on 
forming a passivation layer via electrostatic adsorption using cationic polymer. However, the existing approaches have a limitation in preventing the tungsten dissolution because it is easily torn off by mechanical friction during the CMP process, and as a result, improving topography through modulating tungsten dissolution is insufficient.

In our previous study, the selective adsorption mechanism of pyridine functional groups from the aspect of bonding orbital theory was suggested in copper barrier CMP [11]. Herein, we extended it to the tungsten application using $\alpha$-picolinic acid as an inhibitor to prevent tungsten dissolution, and its impact on tungsten topography was discussed. The pyridine group of picolinic acid plays a role in a ligand forming a coordinate covalent bond with a tungsten atom, resulting in the protection of the surface tungsten atoms from dissolution during the CMP process [12-15]. In an adsorption isotherm experiment, the adsorption amount of picolinic acid adsorbed on tungsten oxide was shown to increase with increasing concentration of picolinic acid. CMP and a dipping test were conducted as a function of picolinic acid concentration. The tungsten surfaces were subsequently analyzed by AFM and SEM.

\section{Materials and Methods}

\subsection{Materials}

About $50 \mathrm{~nm}$ colloidal $\mathrm{SiO}_{2}$ was used as an abrasive. The solid concentration of $\mathrm{SiO}_{2}$ was $3.0 \mathrm{wt} \%$. An amount of $30 \mathrm{wt} \%$ of hydrogen peroxide $\left(\mathrm{H}_{2} \mathrm{O}_{2}\right.$, Daejung Chemical, Siheung-si, Korea) stock solution was used as an oxidizer, and all experiments were carried out after dilution to $4.0 \mathrm{wt} \%$. The concentration of picolinic acid (Sigma Aldrich, Burlington, MA, USA) in the experiment was added to each solution relative to the solution weight. The $\mathrm{pH}$ of the slurries was adjusted to 2.5 using nitric acid $\left(\mathrm{HNO}_{3}, 1.0 \mathrm{~N}\right.$, Sigma Aldrich, Burlington, MA, USA). The $300 \mathrm{~mm}$ CVD deposited tungsten wafers were purchased from Advantec Korea Co., Ltd., Seoul, Korea. These wafers were diced into $6 \times 6 \mathrm{~cm}^{2}$ coupons for polishing experiments.

\subsection{Characterization}

The hydrodynamic diameters of the slurries were measured by dynamic light scattering (DLS, Nano-ZS, Malvern, UK). To describe the effect of picolinic acid on tungsten surface, three-dimensional microscopy was carried out using an atomic force microscope (AFM, XE-150, Park systems, Suwon, Korea) in non-contact mode with a $3 \times 3 \mu \mathrm{m}^{2}$ scan size and a $0.3 \mathrm{~Hz}$ scan rate. Surface images of wafers after polishing were analyzed by a scanning electron microscope (SEM, JSM-7600F, JEOL, Tokyo, Japan).

\subsection{Adsorption Isotherm}

Adsorption behaviors of picolinic acid on tungsten oxide were measured using the solution-depletion method using a total organic carbon analyzer (TOC-5000A, Shimadzu Corp., Kyoto, Japan). We used tungsten oxide in solid powder form ( $<100 \mathrm{~nm}$, Sigma Aldrich, Burlington, MA, USA) because tungsten is oxidized to tungsten oxide by hydrogen peroxide during the actual CMP process. Tungsten oxide slurries were prepared with different concentrations of picolinic acid. The $\mathrm{pH}$ of the slurries was adjusted to 2.5 using nitric acid. Slurries were aged for $12 \mathrm{~h}$ at room temperature with mixing. Centrifugation was carried out to acquire the supernatant to analyze un-adsorbed picolinic acid using TOC analysis and measurement.

\subsection{CMP and SER Evaluation}

A coupon CMP (POLI-300, G\&P Technology, Busan, Korea) equipment with a commercial pad (IC 1010/Suba IV, Dupont, DE, USA) was used for the CMP evaluation. Detailed CMP experimental conditions are shown in Table S1. Static etch rate (SER) experiments used to verify the dissolution rate of tungsten were performed. To measure the SER, tungsten wafer samples cut into $2 \times 2 \mathrm{~cm}^{2}$ in each experimental composition were immersed 3 min in the sufficient amount of slurry solution. The thickness of tungsten films was mea- 
sured using a four-point probe (FFP) (CMT-SR5000, Changmin Tech, Seongnam, Korea). The specific resistance $(\rho)$ of tungsten films was estimated to $5.6 \times 10^{-8} \Omega \cdot \mathrm{m}$, and the thickness was calculated from dividing by the sheet resistance estimated through FFP in a constant condition as shown in the following equation.

$$
\text { Thickness }(\mathrm{cm})=\frac{\text { Specific resistance }(\mathrm{ohm} \cdot \mathrm{cm})}{\text { Sheet resistance }(\mathrm{ohm} / \mathrm{sq})}
$$

The CMP evaluation to obtain material removal rate of tungsten films was repeated three times, and the average numbers are reported here.

\section{Results and Discussion}

The structure of picolinic acid and the schematic illustration of picolinic acid adsorption is shown in Figure 1a,b, respectively. The lone pair electrons in the nitrogen of the pyridine functional group of picolinic acid act as active sites, which leads to the formation of complexes by covalent coordination bonding (i.e., $\sigma$-bonding) with tungsten oxide. Such complexes form a passivation layer on the metal surface, preventing corrosion reactions by blocking the water adsorption $[14,16,17]$. An adsorption isotherm test was carried out to identify the detailed adsorption behavior of picolinic acid on tungsten oxide. Figure 1 $\mathrm{c}, \mathrm{d}$ shows the adsorption isotherm of picolinic acid on tungsten oxide as a function of picolinic acid concentrations. The adsorption data were analyzed using the Langmuir and Freundlich models [18,19].

(a)
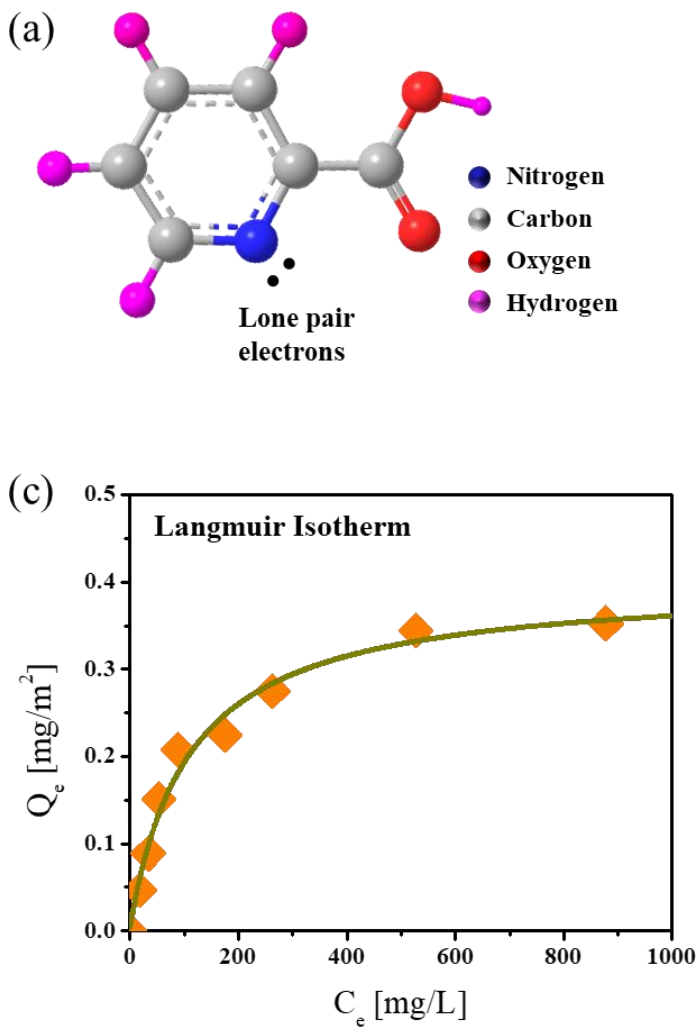

(b)
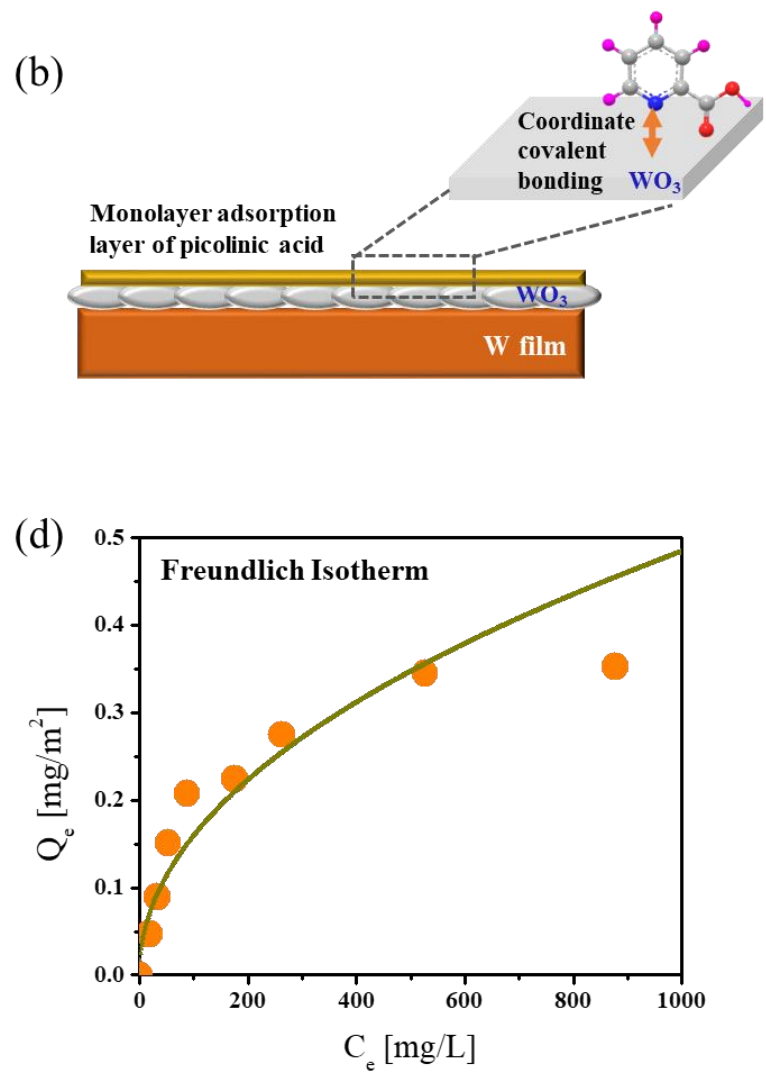

Figure 1. (a) Molecular structure of picolinic acid. (b) Schematic illustration of picolinic acid adsorption onto the tungsten films. Adsorption isotherms of tungsten oxide as a function of picolinic acid concentration analyzed using (c) Langmuir and (d) Freundlich equations. 
The Langmuir model can be described with the equation:

$$
\frac{C_{e}}{Q_{m}}+\frac{1}{b Q_{m}}=\frac{C_{e}}{Q_{e}}
$$

and the Freundlich model can be expressed as:

$$
Q_{e}=K_{F} \cdot C_{e}{ }^{1 / n}
$$

where $Q_{e}$ is the concentration of adsorbed picolinic acid on the surface of tungsten oxide $\left(\mathrm{mg} / \mathrm{m}^{2}\right), Q_{m}$ is the maximum concentration of adsorbed picolinic acid $\left(\mathrm{mg} / \mathrm{m}^{2}\right), C_{e}$ is the concentration of picolinic acid in slurry solution $(\mathrm{mg} / \mathrm{L})$, and $b$ is the Langmuir constant $(\mathrm{L} / \mathrm{mg})$ [18]. The Freundlich constant $K_{F}$ is related to the adsorption capacity, and $1 / n$ is correlated the adsorption intensity [19].

The constants calculated from adsorption models are shown in Table 1 . The higher $\mathrm{R}^{2}$ value of the Langmuir model compared to that of the Freundlich model indicates that the picolinic acid was homogeneously adsorbed on tungsten oxide [18,19], meaning that the picolinic acid forms a monolayer on the surface of tungsten oxide [18]. Both Lee et al. and Ramis et al. experimentally demonstrated that the strong Lewis acid sites and Brønsted acid sites on the surface of metal oxide films could be active sites for the adsorption of pyridine functional groups $[11,20,21]$. From this point of view, the coordinated covalent bonding between the pyridine group of picolinic acid and the acidic sites of tungsten oxide resulted in monolayer adsorption on the surface of tungsten oxide [12,13,20,21]. Based on adsorption isotherm results, the saturated concentration of picolinic acid was determined to be $1.5 \mathrm{wt} \%$. Thus, we selected four picolinic acid concentrations $(0,0.5,1.5$, and $5.0 \mathrm{wt} \%$ per solution weight) for subsequent experiments to determine its effect on tungsten dissolution.

Table 1. Langmuir and Freundlich constants for the adsorption of picolinic acid onto the tungsten oxide surface.

\begin{tabular}{cccccc}
\hline \multicolumn{3}{c}{ Langmuir Constant } & \multicolumn{3}{c}{ Freundlich Constant } \\
\hline $\mathbf{Q}_{\mathbf{m}}\left[\mathbf{m g} / \mathbf{m}^{2}\right]$ & $\mathbf{b}[\mathbf{L} / \mathbf{m g}]$ & $\mathbf{R}^{\mathbf{2}}$ & $\mathbf{K}_{\mathbf{F}}$ & $\mathbf{1 / n}$ & $\mathbf{R}^{\mathbf{2}}$ \\
\hline 0.399 & 0.009 & 0.994 & 0.017 & 0.479 & 0.825 \\
\hline
\end{tabular}

To verify the dispersion stability of the silica nanoparticles with picolinic acid as a CMP slurry, we measured the hydrodynamic diameters and zeta potentials of the slurries as a function of the picolinic acid concentration, as shown in Figure S1 and Table S2. As the concentration of picolinic acid increased, the measured hydrodynamic diameters was maintained at the level of $64 \mathrm{~nm}$ and did not change. Similarly, the zeta potentials of the slurries also constantly remained at the level of $-20 \mathrm{mV}$ as the concentration of picolinic acid increased. These results confirm that the dispersion stability of the silica-based slurry is not influenced by the addition of picolinic acid.

After the dipping test, the topography was analyzed by AFM and SEM, as seen in Figure 2. The average roughness $\left(R_{a}\right)$ of each sample was calculated to quantitatively compare their topographies. In the absence of picolinic acid, the grains of tungsten were severely damaged due to the high dissolution rate of tungsten. Similar results were obtained with $0.5 \mathrm{wt} \%$ picolinic acid, but a few more grains were observed. When 1.5 and $5.0 \mathrm{wt} \%$ picolinic acid were added to the slurry, the grains of tungsten remained well because the amounts of adsorbed picolinic acids were sufficient to suppress tungsten dissolution. In general, corrosion (i.e., dissolution) of tungsten mainly occurs at the grain boundary with high thermodynamic activation energy. However, since picolinic acid protects the reaction active site via adsorbing to the tungsten grain boundary through covalent coordination bonding, the surface roughness could be dramatically improved. The $R_{a}$ values obtained from AFM and SEM images were well correlated. 
(a)

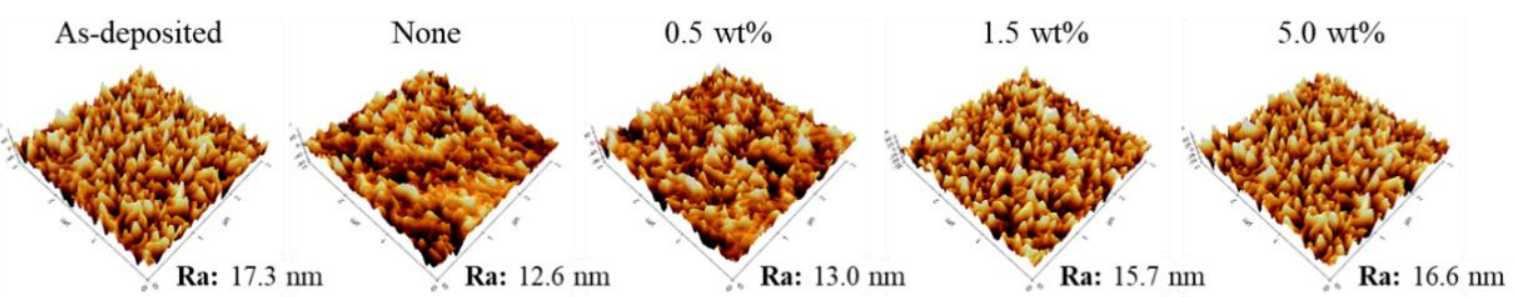

(b)
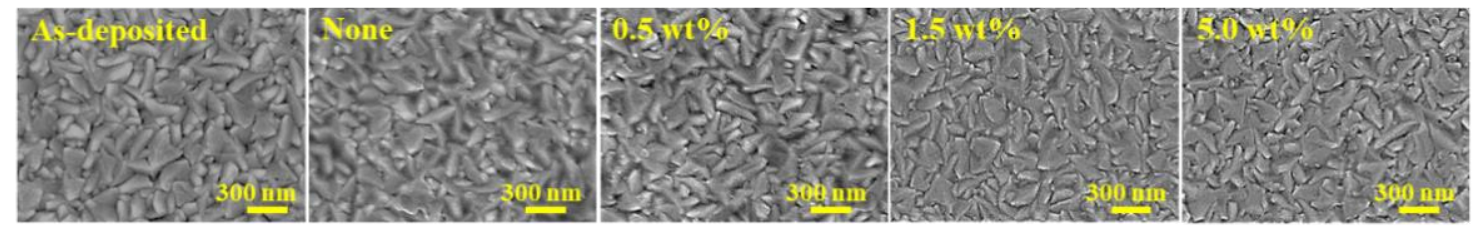

Figure 2. (a) Atomic force microscopy (AFM) and (b) scanning electron microscope (SEM) images of tungsten films after the dipping test as a function of picolinic acid concentration.

As shown in Figure 3, the surface of tungsten films after the CMP test were analyzed by AFM and SEM. In the absence of picolinic acid, grains remained despite the application of CMP process. The persistence of the grains result from dissolution in the valleys between the grains as the tops are polished. Using $0.5 \mathrm{wt} \%$ of picolinic acid failed to remove the grains, as the picolinic concentration was not sufficient to prevent dissolution in the tungsten valleys. On the other hand, the grains were well removed with $1.5 \mathrm{wt} \%$ picolinic acid, and $\mathrm{R}_{\mathrm{a}}$ decreased from $17.3 \mathrm{~nm}$ to $7.8 \mathrm{~nm}$ after CMP process. This result indicates that $1.5 \mathrm{wt} \%$ picolinic acid is sufficient for the protection of the tungsten valleys between the grains during the CMP such that the topography was smoothed.

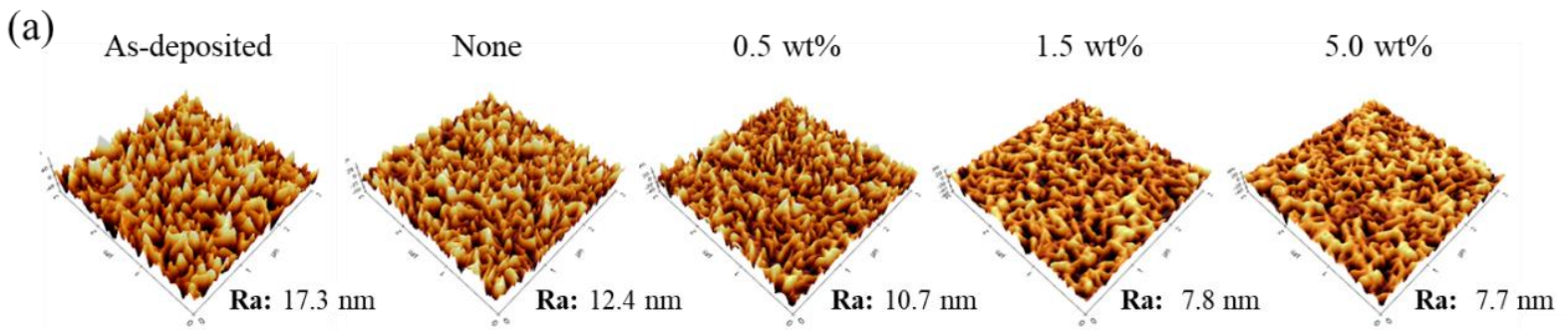

(b)

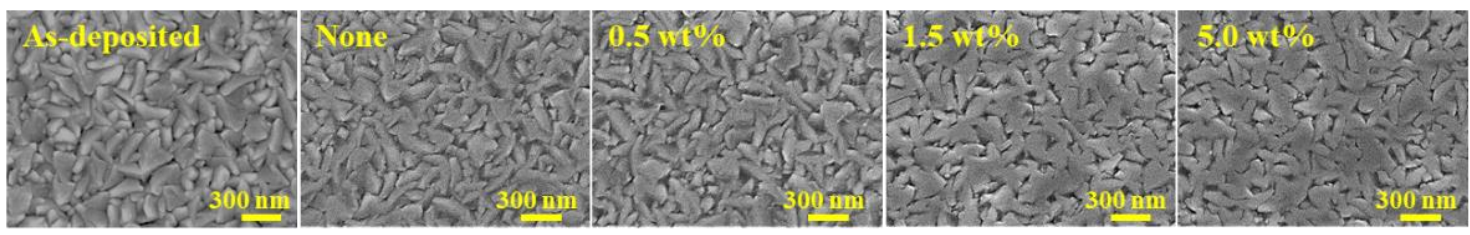

Figure 3. (a) Atomic force microscopy (AFM) and (b) scanning electron microscope (SEM) images of tungsten films after the CMP evaluation as a function of picolinic acid concentration.

The removal and dissolution rates of tungsten as a function of picolinic acid concentration were calculated and are shown in Figure 4. The removal rate of the tungsten films gradually decreased from 120 to $85 \AA$ /min with increasing concentration of picolinic acid up to $1.5 \mathrm{wt} \%$ due to the adsorption of picolinic acid. Additionally, at this picolinic acid concentration, the topography of tungsten, as represented by the $R_{a}$ value, was well removed. On the other hand, the tungsten dissolution rate dramatically decreased from 90 to 
$11 \AA /$ min as picolinic acid concentration increased up to $1.5 \mathrm{wt} \%$, while a high $R_{a}$ value was maintained. This result indicates that the excessive tungsten dissolution was suppressed. Both the removal and dissolution rates decreased up to a picolinic acid concentration of $1.5 \mathrm{wt} \%$ and showed almost the same results at $5.0 \mathrm{wt} \%$ because the adsorption of picolinic acid onto the surface of tungsten oxide saturates at this concentration. These results are in good agreement with the adsorption isotherm simulations.

(a)

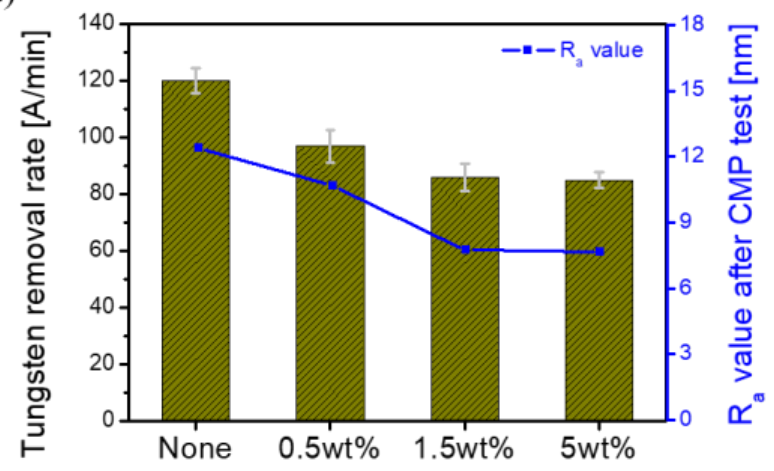

(b)

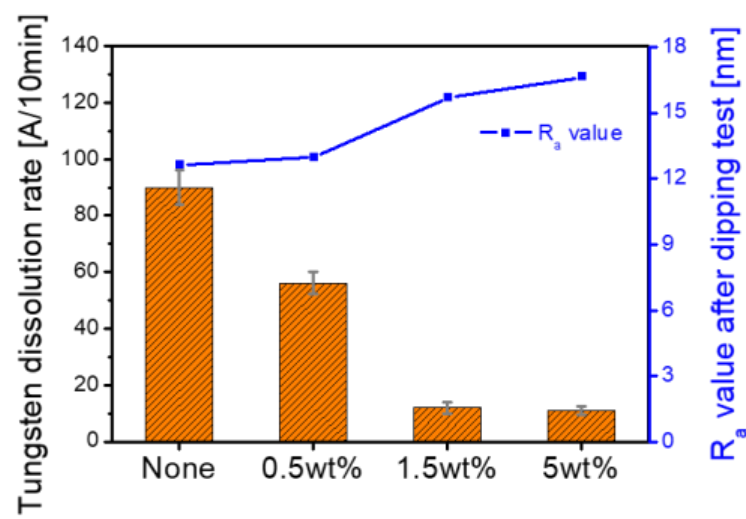

Figure 4. (a) Dissolution rates and $R_{a}$ values from the tungsten dipping test and (b) removal rates and $\mathrm{R}_{\mathrm{a}}$ values from the tungsten CMP test for various picolinic acid concentrations.

\section{Conclusions}

In order to remove the topography of the tungsten surface during the CMP process, the effect of picolinic acid on tungsten dissolution was studied. First, the adsorption behavior of picolinic acid on tungsten oxide was analyzed using the Langmuir and Freundlich model equations. The adsorption of picolinic acid on tungsten oxide fit well with the Langmuir model and saturated at $1.5 \mathrm{wt} \%$. The removal and dissolution rates were tested at selected picolinic acid concentrations and were shown to decrease with picolinic acid concentration until saturation at $1.5 \mathrm{wt} \%$, which is in good agreement with the adsorption behavior. To analyze the surface topography of each sample, AFM and SEM were used. Tungsten grains were well removed with $1.5 \mathrm{wt} \%$ picolinic acid because the valleys of tungsten did not dissolve due to the adsorption of picolinic acid, while the tops were polished until the entire surface flattens, resulting in minimizing the tungsten topography below $10 \mathrm{~nm}$. The experimental results in this study are expected to play a role in ameliorating defects and enhancing the buffing efficiency during the tungsten CMP process for next generation semiconductor process.

Supplementary Materials: The following supporting information can be downloaded at: https: / / www.mdpi.com/article/10.3390/app12031227/s1, Table S1: Detailed CMP process conditions; Figure S1: Hydrodynamic diameters of $\mathrm{SiO}_{2}$ abrasive as a function of the concentration of picolinic acid; Table S2: Hydrodynamic diameters and Zeta potential of $\mathrm{SiO}_{2}$ abrasive as a function of the concentration of picolinic acid.

Author Contributions: Conceptualization, K.L.; methodology, K.L.; investigation, K.L. and J.S.; data curation, K.L.; writing—original draft preparation, K.L.; writing—review and editing, K.L. and J.S. All authors have read and agreed to the published version of the manuscript.

Funding: This research received no external funding.

Institutional Review Board Statement: Not applicable.

Informed Consent Statement: Not applicable.

Data Availability Statement: Not applicable.

Conflicts of Interest: The authors declare no conflict of interest. 


\section{References}

1. Wang, G.; Xu, Q.; Yang, T.; Xiang, J.; Xu, J.; Gao, J.; Li, C.; Li, J.; Yan, J.; Chen, D. Application of atomic layer deposition tungsten (ALD W) as gate filling metal for $22 \mathrm{~nm}$ and beyond nodes CMOS technology. ECS J. Solid State Sci. Technol. 2014, 3 , P82. [CrossRef]

2. Lee, P.I.; Cronin, J.; Kaanta, C. Chemical vapor deposition of tungsten (CVD W) as submicron interconnection and via stud. J. Electrochem. Soc. 1989, 136, 2108. [CrossRef]

3. Tsao, S.; Blewer, R.; Tsao, J. Tungsten deposition on porous silicon for formation of buried conductors in single crystal silicon. Appl. Phys. Lett. 1986, 49, 403-405. [CrossRef]

4. Lim, G.; Lee, J.-H.; Kim, J.; Lee, H.-W.; Hyun, S.-H. Effects of oxidants on the removal of tungsten in CMP process. Wear 2004, 257, 863-868. [CrossRef]

5. Seo, J.; You, K.; Moon, J.; Kim, J.H.; Paik, U. Communication-Corrosion behavior of tungsten metal gate in the presence of hydrogen peroxide at acidic medium. ECS J. Solid State Sci. Technol. 2017, 6, P169. [CrossRef]

6. Kaufman, F.; Thompson, D.; Broadie, R.; Jaso, M.; Guthrie, W.; Pearson, D.; Small, M. Chemical-mechanical polishing for fabricating patterned W metal features as chip interconnects. J. Electrochem. Soc. 1991, 138, 3460. [CrossRef]

7. Lee, K.; Seo, J.; Paik, U. Preparation and characterization of slurry for CMP. In Advances in Chemical Mechanical Planarization (CMP); Elsevier: Amsterdam, The Netherlands, 2022; pp. 323-354.

8. Yu, H.; Wang, X.; Sheng, H.; Lu, W.; Zhou, M. Local-Topography-Induced Defects during Tungsten Chemical Mechanical Polishing and Their Impact on Back End of Line. J. Electrochem. Soc. 2008, 156, H64. [CrossRef]

9. Kanner, G.S.; Butt, D.P. Raman and electrochemical probes of the dissolution kinetics of tungsten in hydrogen peroxide. J. Phys. Chem. A 1998, 102, 9501-9507. [CrossRef]

10. Anik, M.; Cansizoglu, T. Dissolution kinetics of WO 3 in acidic solutions. J. Appl. Electrochem. 2006, 36, 603-608. [CrossRef]

11. Lee, K.; Sun, S.; Lee, G.; Yoon, G.; Kim, D.; Hwang, J.; Jeong, H.; Song, T.; Paik, U. Galvanic corrosion inhibition from aspect of bonding orbital theory in Cu/Ru barrier CMP. Sci. Rep. 2021, 11, 21214. [CrossRef] [PubMed]

12. Angelici, R.J.; Malone, S.M.D. Infrared studies of amine, pyridine, and phosphine derivatives of tungsten hexacarbonyl. Inorg. Chem. 1967, 6, 1731-1736. [CrossRef]

13. Johnson, J.W.; Jacobson, A.; Rich, S.; Brody, J. New layered compounds with transition-metal oxide layers separated by covalently bound organic ligands. Molybdenum and tungsten trioxide-pyridine. J. Am. Chem. Soc. 1981, 103, 5246-5247. [CrossRef]

14. Bleeke, J.R. Metallabenzenes. Chem. Rev. 2001, 101, 1205-1228. [CrossRef] [PubMed]

15. Huang, X.; Zhai, H.J.; Kiran, B.; Wang, L.S. Observation of d-orbital aromaticity. Angew. Chem. Int. Ed. 2005, 44, 7251-7254. [CrossRef] [PubMed]

16. Seo, J. A review on chemical and mechanical phenomena at the wafer interface during chemical mechanical planarization. J. Mater. Res. 2021, 1-23. [CrossRef]

17. Seo, J.; Vegi, S.H.; Ranaweera, C.; Baradanahalli, N.; Han, J.-H.; Koli, D.; Babu, S. Formation of cobalt-BTA complexes and their removal from various surfaces relevant to cobalt interconnect applications. ECS J. Solid State Sci. Technol. 2018, 8, P3009. [CrossRef]

18. Langmuir, I. The constitution and fundamental properties of solids and liquids. Part I. Solids. J. Am. Chem. Soc. 1916, 38, 2221-2295. [CrossRef]

19. Freundlich, H. Über die adsorption in lösungen. Z. Für Phys. Chem. 1907, 57, 385-470. [CrossRef]

20. Ramis, G.; Cristiani, C.; Elmi, A.S.; Villa, P.; Busca, G. Characterization of the surface properties of polycrystalune WO3. J. Mol. Catal. 1990, 61, 319-331. [CrossRef]

21. Ramis, G.; Busca, G.; Cristiani, C.; Lietti, L.; Forzatti, P.; Bregani, F. Characterization of tungsta-titania catalysts. Langmuir 1992, 8, 1744-1749. [CrossRef] 